\title{
The Space Bender: Supporting Natural Walking via Overt Manipulation of the Virtual Environment
}

\author{
Adalberto L. Simeone* \\ KU Leuven, Belgium \\ Niels Christian Nilsson ${ }^{\dagger}$ \\ Aalborg University Copenhagen, Denmark \\ Marco Speicher $\$$ \\ Florian Daiber ${ }^{\text {II }}$ \\ DHfPG, Saarbrücken, Germany \\ DFKI, Saarbrücken, Germany
}

\begin{abstract}
Manipulating the appearance of a Virtual Environment to enable natural walking has so far focused on modifications that are intended to be unnoticed by users. In our research, we took a radically different approach by embracing the overt nature of the change. To explore this method, we designed the Space Bender, a natural walking technique for room-scale VR. It builds on the idea of overtly manipulating the Virtual Environment by "bending" the geometry whenever the user comes in proximity of a physical boundary. Our aim was to evaluate the feasibility of this approach in terms of performance and subjective feedback. We compared the Space Bender to two other similarly situated techniques: Stop and Reset and Teleportation, in a task requiring participants to traverse a $100 \mathrm{~m}$ path. Results show that the Space Bender was significantly faster than Stop and Reset, and preferred to the Teleportation technique, highlighting the potential of overt manipulation to facilitate natural walking.
\end{abstract}

Index Terms: Human-centered computing-Interaction Paradigms—Virtual Reality;

\section{INTRODUCTION}

Natural walking in a Virtual Environment (VE) is one of the quintessential components of egocentric Virtual Reality (VR) experiences. However, VEs are often much larger than the physical environment in which the user is actually located. To address this problem, various locomotion techniques have been proposed [20].

Redirected walking techniques manipulate users' physical path to keep them within the available tracking space. Approaches to redirected walking can be grouped in two main categories [19]: techniques that manipulate the mapping between users' real and virtual movements [22], and techniques that manipulate the geometry of the VE [30]. A considerable advantage of redirected walking is that the user is physically walking and therefore experiences more accurate proprioceptive, kinesthetic, and vestibular sensations [19]. However, many techniques are likely to be noticed by the user, if the walkable area is not larger than at least $6 \mathrm{~m} \times 6 \mathrm{~m}[1,28]$.

The second category comprises works focusing on the manipulation of the user's spatial perception to make (potentially impossible) architectural changes to the VE [30,31]. Their application however requires specific types of VEs, such as indoor room-based layouts. Techniques that manipulate the VE have so far focused on hidden changes with the aim of understanding the detection thresholds and their effectiveness. For example: the manipulation of doorways in

\footnotetext{
*adalberto.simeone@kuleuven.be

†ncn@create.aau.dk

† andre.zenner@dfki.de

§m-speicher@dhfpg-bsa.de

Iflorian.daiber@dfki.de
}

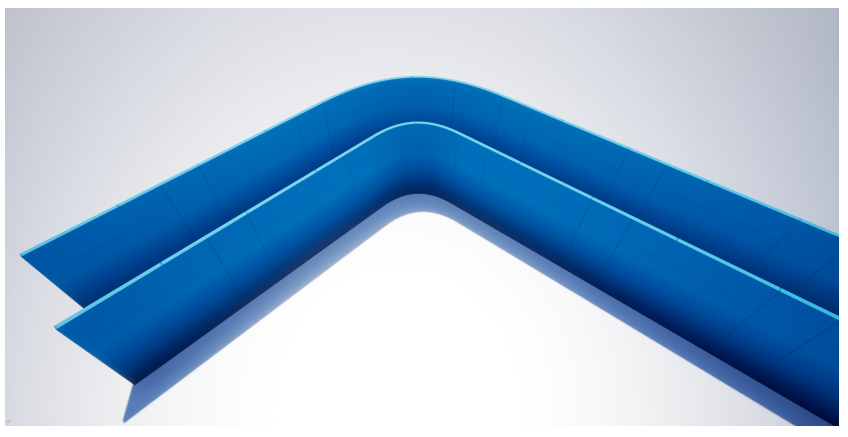

Figure 1: The Space Bender technique "bends" the VE when users are close to physical boundaries, to allow them to continue walking.

such a way that users exit a room from a different direction than the one they entered with, thereby reusing the space available [30]; overlapping adjacent rooms [31]; generating dynamic corridors that connect rooms by leveraging the two previous approaches [37].

Our contribution is the design and evaluation of a novel VE manipulation technique that takes a radically different approach to support locomotion. The Space Bender, as its name suggests, is based on the idea of "bending" the geometry of the VE to follow the layout of the physical room. In doing so, it presents various advantages. Firstly, it leaves any spatial constraint in the VE unchanged. The changes to the VE are temporary and are applied via a collision trigger only when the user is in proximity of a physical boundary. The VE is restored to its unbent state once the user traverses the bend, as opposed to a constant warping [32]. Secondly, the technique leaves users free to interact with the environment, whereas resetting techniques suited for room-scale VR either require the use of the controller to explicitly apply the redirection $[7,40]$ or teleport the user [4], or explicit interaction between the user and an external operator to reorient the user [39]. Finally, it does not require external elements such as distractors [21], in order to perform the redirection, which might be not contextually appropriate or become repetitive when applied to small spaces.

We tested the technique on a simple but not trivial scenario: traversing a $100 \mathrm{~m}$ corridor. We identified two other techniques that are especially suited to similar room-scale scenarios: the Stop and Reset technique [7] and the stock Teleportation implementation of the Unreal Engine. In evaluating the Space Bender technique we wanted to answer the following two research questions: 1) how effective is the technique from both the quantitative (i.e. task completion times, walking speed, drifting) and the qualitative perspective (cybersickness and user preference)? 2) does the overt manipulation of the VE constitute a break in Presence too great to be ignored?

We hypothesized that 1) the Space Bender would lead to faster completion times, as a major drawback of resetting techniques is the necessity to come to a full stop each time the user reaches a boundary; 2) it would be preferred to the other two alternatives as 
the method of choice in similar locomotion scenarios. The results of a within-subject study involving 19 participants confirmed that the Space Bender technique was the best performing walking technique. It led users to walk significantly faster and reach the exit in significantly less time than with the Stop and Reset, and was chosen by 11 of the 19 participants $(57 \%)$ as their most preferred technique.

\section{Related Work}

Generally, redirection techniques can be divided into two broad categories: those that manipulate the physical path of walking users (redirected walking) and those that provide users with an alternative means of travelling to other locations in the VE that can then be explored by walking (relocation techniques) [19]. In the following, we describe those techniques that are closely related to our work, and the relatively small collection of novel approaches, to which the Space Bender belongs, that rely on the manipulation of the VE rather than of the mappings between real and virtual movement.

\subsection{Redirected Walking}

Redirected walking was originally proposed by Razzaque et al. [22] who enabled users to explore a VE while remaining within a comparatively small tracking space by applying imperceptible rotation gains (i.e. manipulation of the mapping between the users real and virtual rotations). Since then, a large body of research has explored similar and novel ways of manipulating users' viewpoint through application of translation [11,38], rotation [5,12], curvature $[9,18,28]$, and bending gains $[14,15]$.

Bending gains is among the approaches able to compress the largest VE into a living-room sized tracking space. When users are walking along a predefined curved virtual path, the user's physical path can be bent by up to 4.4 times its real radius [15], and using bending gains, a VE of about $25 \mathrm{~m} \times 25 \mathrm{~m}$ has been presented within a physical area of about $4 \mathrm{~m} \times 4 \mathrm{~m}$ [14]. Rietzler et al. found that gains up to $20 \% \mathrm{~m}$ will be noticed but still accepted by users [23].

While overt redirected walking has been used to compress VEs into comparatively small tracking spaces (e.g., Seven League Boots [11]), the most common application of overt approaches is resetting controllers [19] - a combination of interventions preventing users from exceeding the bounds of the walkable area and overt redirection guiding the user back toward the centre of this area. Williams et al. [39] proposed three different resetting controllers. FreezeBackup resetting where the system intervenes by freezing the virtual experience, which remains frozen until users have been guided to the centre of the walkable area. Freeze-Turn resetting where a similar intervention is used but users turn toward the centre of the walkable area before the experience is resumed. Finally, with 2:1 Turn resetting the system intervenes by asking users to turn on the spot, and while turning the rotation is scaled so that a $180^{\circ}$ physical rotation results in a $360^{\circ}$ virtual rotation. This leaves the user physically facing the centre of the walkable area while their virtual orientation is the same as before the turn.

In our previous work, we introudced the Stop and Reset technique [7], which uses collision between the controller and a distractor to virtually rotate the user $180^{\circ}$ in place. The technique is itself inspired by the previously described Freeze-Turn technique [39] and the Bookshelf technique [40], which uses an actual bookshelf that once activated via a button proceeds to virtually rotate the platform on which it is located, along with the user, by $180^{\circ}$.

\subsection{Relocation Techniques}

The design space of Relocation Techniques ranges from techniques mimicking real-world vehicles to magical travel [29]. Here we only cover those approaches that resemble the magical relocation technique included in the current study, i.e. the Teleportation technique.

Bolte et al. [2] proposed the Jumper metaphor that combines real walking and teleportation for covering short and long distances, respectively. Høeg et al. [10] found that button clicks appear to require the least explicit attention and it was experienced as less disorienting, less physically demanding, and more enjoyable. The teleportation technique described by Bozgeyikli et al. [4] decouples target selection from the viewing direction and the target is instead chosen by pointing. Frommel et al. [8] explored teleportation to fixed locations, finding that the ability to freely choose the target destination elicited lower disorientation and higher presence. Langbehn et al. [16] compared joystick navigation, redirected walking based on bending gains, and teleportation where the target is selected by pointing and teleportation instigated by pressing a button. The results indicated that redirected walking may be superior with respect to spatial knowledge acquisition, and redirected walking and teleportation was preferred over joystick navigation which also led to the most motion sickness.

Because teleportation can be disorienting [3], researchers have experimented with magical metaphors as a basis for teleportation [29]. Steinicke et al. [27] introduced virtual portals as a means of transporting users from a replica of the physical laboratory to the $\mathrm{VE}$, and found that it positively influenced the users' sensation of presence and their ability to estimate virtual distances.

\subsection{Manipulation of the Virtual Environment}

The first approach relying on manipulation of the VE was proposed by Suma et al. [30] who showed that users' physical paths can be manipulated by subtly changing the placement of doors and corridors, when users were not looking. Using this approach, dubbed Change Blindness Redirection, Suma et al. [30] were able to present a virtual office building of about $219 \mathrm{~m}^{2}$ within a tracking space of $4.3 \mathrm{~m} \times 4.3 \mathrm{~m}$.

Suma et al. [31] empirically demonstrated that adjacent virtual rooms can overlap considerably without the user noticing it (i.e. relatively small virtual rooms may overlap by up to $56 \%$ and larger virtual rooms by up to $31 \%$ ). Notably, participants appeared to perceive distances to objects in the adjacent rooms as if no overlap was present, even when the manipulation was overt. Moreover, studies suggest that noticeability can be decreased and the degree of overlap increased by using longer corridors with additional corners [35], and corridors with smooth curves, rather than right angles, may be more beneficial for spatial compression [36]. Finally, the algorithm Flexible Spaces is able to procedurally generate large, but not necessarily plausible, interior VEs within a given walkable area by combining the principles of Impossible Spaces and Change Blindness Redirection [37].

Sun et al. [32] proposed a radically different approach to redirection based on manipulation of the appearance of the VE. It fits VEs into comparatively smaller tracking spaces by computing a planar map between the real and virtual environments and re-projecting the warped environment in a way that ensures an acceptable level of visual comfort. While in this approach the VE is experienced with distortions applied, in the Space Bender they are only visible when the user approaches the boundary of the walkable area, and restored immediately after the user leaves the manipulated area.

\section{Techniques}

In this section we describe the design and implementation of the Space Bender technique. We also report the details of the implementation of the two other techniques considered in the user study: an adaptation of the Stop and Reset technique originally proposed by Cools and Simeone, and the implementation of the Teleportation technique provided by the Unreal Engine. All three techniques were evaluated on a task requiring participants to traverse a $100 \mathrm{~m}$ path.

\subsection{The Space Bender Technique}

The Space Bender technique attempts to provide a seamless locomotion experience in VEs that are larger than the physical space 

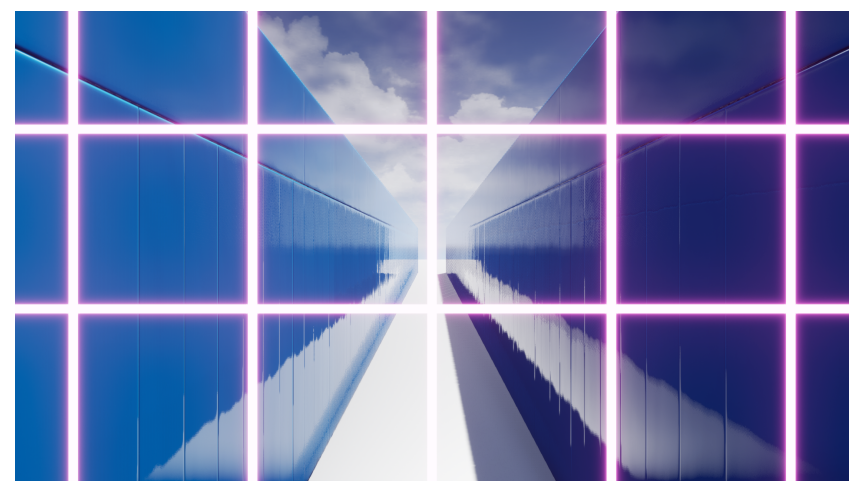

Figure 2: The figure shows how the Stop and Reset would have looked like with the Chaperone Grid visible.

available. Its core concept is, as its name suggests, the idea of bending the geometry of the VE to accommodate the layout of the physical environment (see Figures 1 and 5). The technique relies on a compromise: by accepting the overtness of the manipulation, it allows users to walk across large distances.

We leveraged a feature of 3D game engines: spline meshes (in Unreal Engine 4 terminology; also known as deformable meshes - a free Unity plugin is also available ${ }^{1}$ ). These are $3 \mathrm{D}$ assets with enough vertices to be deformed along a spline curve. For example, a wall asset having just eight vertices will not be able to show any curvature as there are no vertices along the sides that can be moved to follow the shape of the curve. In our implementation, we used 64 vertices per metre of wall to get a detailed approximation.

The proof of concept system we built consists of a procedural generation algorithm to create an abstract VE consisting of the $100 \mathrm{~m}$ path, and of the "bending" logic applied to each bendable segment of the path. The procedural generation system replicates a user-defined $3 \mathrm{D}$ asset as many times as needed to create a path of the desired length. We used two $1 \mathrm{~m} \times 3 \mathrm{~m} \times 10 \mathrm{~cm}(l \times h \times d)$ wall assets to delimit the path. However any asset, from an outdoor flat trail to a walkway, could have been used. In our previous research, we found that users will follow paths indicated by virtual obstacles [24]. The system then retrieves the bounds of the area the user delimited during setup (the Chaperone Area, see Fig. 5), and uses it to determines the maximum length of each segment of the path. The algorithm then creates a sequence of segments up to the desired length.

In our implementation, each segment of the path (measuring $350 \mathrm{~cm}$ ) consists of a spline mesh. The length was chosen in order to fit four segments in the physical area available of $5 \mathrm{~m} \times 5 \mathrm{~m}$. When a segment is bent, its shape is calculated via a quadratic Bezier, using as start and end points those of the previous and successive segments (if any). Since the calculation of the offset or parallel curve is a nontrivial problem, we used a numerical solution ${ }^{2}$. When the user is not close to the boundaries of the play area, the VE appears unaltered (see Fig. 2). In our case, the virtual corridor appeared completely straight. The collision trigger is placed at a distance of ca. $1.17 \mathrm{~m}$, in the middle of the segment. This length represents the maximum distance that the user can walk before the path needs to be bent. It was based on results from a study by Sreenivasa et al. that indicate that participants start to turn their head towards the direction of the turn $1.10 \mathrm{~m}$ before the obstacle [26].

When the user exits the trigger, an animation (lasting one second) is triggered showing the remainder of the path bending as much as

\footnotetext{
${ }^{1}$ SplineMesh by Benoit Dumas: https://assetstore.unity.com/ packages/tools/modeling/splinemesh-104989

${ }^{2} \mathrm{An}$ implementation is available at https://github.com/AriaXR/ SpaceBender
}

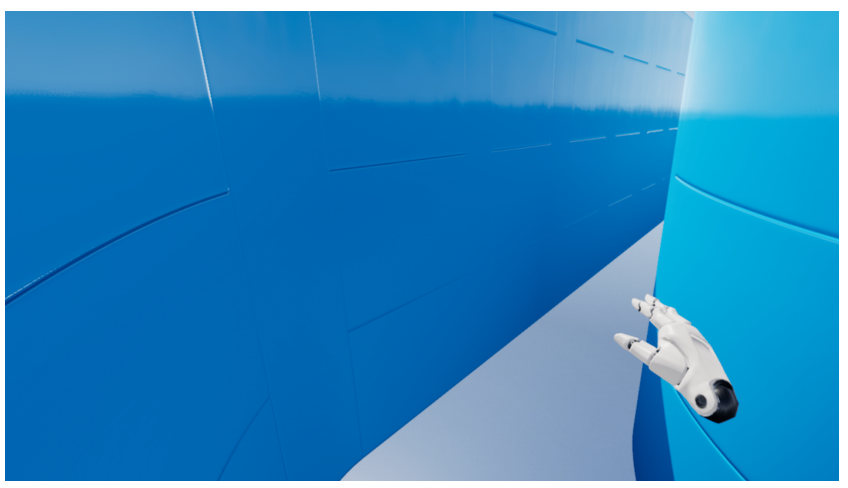

Figure 3: The figure shows a bent section of the path. Once the user will have walked past, it will straighten itself behind them.

needed to follow the layout of the room (in our case, for a square area, a bend of $90^{\circ}$ ). When the bend occurs, the user will have approached a corner or boundary in the physical environment. Once the user follows the bendable segment and leaves the third segment, entering in a new section of the path, the sections behind the users are straightened. If the user were to look behind themselves, they would see a straight path. This change happens when the user enters the next segment (and is thus no longer close to the bend).

\subsection{The Stop and Reset Technique}

The Stop and Reset is an adaptation of the one we implemented in the context of redirection in room-scale spaces via interactive distractors [7], inspired by the previously described Bookshelf technique [40] and the Freeze-Turn technique by Williams et al.

In the original paper by Williams et al., the authors described three resetting techniques [39]. We initially implemented the fastest two: the Freeze-Turn and 2:1 Turn. The description of the algorithm reported in the paper indicates that upon reaching the boundaries of the tracking area "the user turns around with the virtual screen frozen until he or she feels that they have turn approximately $180^{\circ}$ " We ran a pilot test with five users, and according to their feedback and our own observations, turning with the tracking frozen was very likely to cause dizziness and nausea. Likewise, the 2:1 Turn, as it relied on doubling the rotational speed in such a way that a $180^{\circ}$ physical turn would equal to a $360^{\circ}$ in the VE.

In order to have a fairer comparison with the other two techniques, we thus opted to adapt the Stop and Reset variant previously described instead. In our implementation, the user walks normally until they reach a physical boundary. This will trigger the appearance of the Chaperone Grid (since the actual SteamVR grid overlay is only visible in the HMD, Fig. 2 shows a mock-up). Whenever the user presses the trigger button on their controller, the system will effectively teleport them in the same location, but with their orientation in the VE exactly rotated by $180^{\circ}$ from whichever orientation they had before, thus facing the opposite direction. Before the rotation, the user's view fades to black and then fades back in with the new orientation. The duration of this process uses the same values used in the stock Teleportation technique provided by the Unreal Engine, i.e. $300 \mathrm{~ms}$. A successive physical $180^{\circ}$ turn rotates users towards the direction in which they needed to continue walking.

\subsection{Teleportation}

The Teleportation technique was chosen in the comparison as it is the de-facto standard locomotion method used in VR room-scale experiences and does also allow users to walk. We used the implementation provided in the Unreal Engine 4. It works by pressing the controller's trackpad, which displays a parabolic ray. When the user releases the trackpad, they will be teleported in the location indicated 


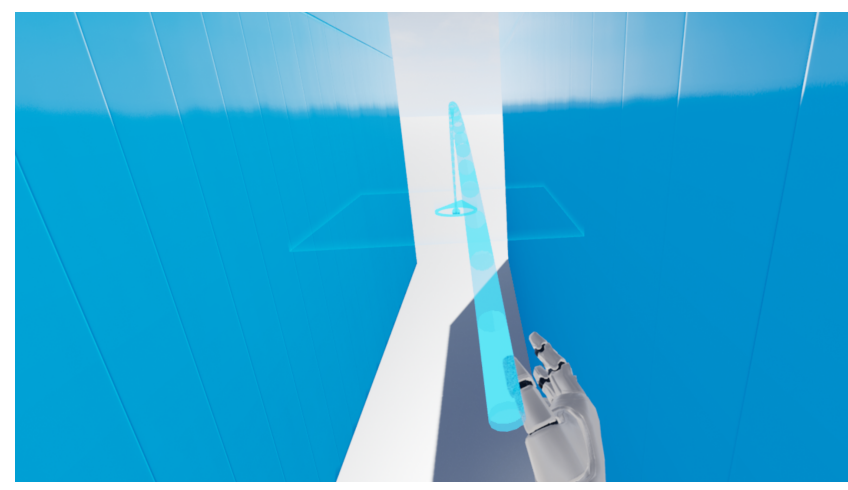

Figure 4: The figure shows the default Teleportation technique as implemented in the Unreal Engine 4.19.2.

by a marker placed where the ray intersects the ground. Users can control the distance at which they will be teleported through the controller's inclination, which is clamped at $10 \mathrm{~m}$. The user can also control the post-teleportation orientation by using the controller's trackpad, indicated by an arrow inside the marker (see Fig. 4).

\section{USER StUdy}

The main aim of the study was to understand whether overtly manipulating the VE constitutes a valid design approach for supporting locomotion. In order to answer the first research question on the performance of this approach, we measured kinematic metrics such as speed, time, and distance walked. Additionally, we measured how much time is spent actually walking and thus not idling, as an indication of the efficiency of the technique.

Lastly, we logged users' real-world trajectories in order to detect whether drifting occurred in the physical environment, to understand how well each technique uses the physical space available. We also asked participant to fill in questionnaires such as the Simulator Sickness Questionnaire (SSQ) [13], the SUS Presence questionnaire [34], and a custom one asking them further questions that were not captured by the first two.

To answer the second research question on whether this type of overt manipulation would interfere too much with the user's experience in VR, after completing the tasks with all three techniques we conducted a semi-structured interview with participants to elicit their feedback and better understand their behaviour.

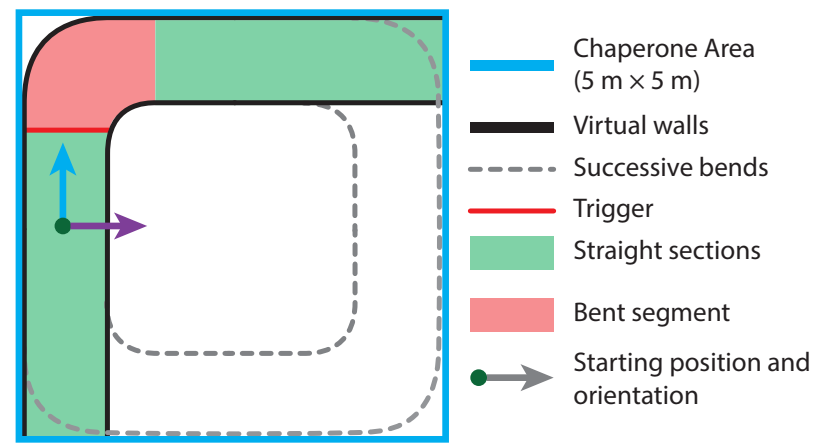

Figure 5: The diagram shows the layout of the room and of the VE when a bend has occurred, and how the successive segments will be bent when the user continues walking.

\subsection{Participants and Apparatus}

We recruited 19 (six females) volunteer participants (aged $20-$ $39, M=28.53, S D=5.43$ ) through online social media advertising. They wore an HTC Vive head-mounted display and carried a single controller throughout each session. In order to avoid the risk of cables hindering their movement, we used TPCast, an add-on that is mounted on top of the HMD's head-strap. It allows wireless operation of the headset and is powered by a $20100 \mathrm{~mA} / \mathrm{h}$ battery carried on a belt worn around their waist.

\subsection{Task}

The task consisted in reaching the exit of a straight path measuring $100 \mathrm{~m}$ using each of the three techniques. We built a minimalistic VE using the Unreal Engine 4.19.2, where two walls delimited the path to follow ( $1 \mathrm{~m}$ wide, see Fig. 4). Due to the abstract nature of the environment, we added a feature that changed the diffuse colour of the material used for the walls from blue to red, as they walked past, and outside their view. We told participants that, should they feel lost, the sections of the path that they had already walked past would be coloured in red.

\subsection{Procedure}

We first welcomed participants and asked them to read the informative document detailing the conditions of the user study, which had been positively assessed by our university's ethics board. Successively, we asked them to fill in their demographics data. We explained that their goal was to reach the exit of the path in front of them, with each of the three techniques. There was no time limit. Before starting the study, we asked participants to walk the length of the room back and forth four times, while wearing the HTC Vive on top of their head (but without looking through it) and holding a Vive controller, to have an indication of their walking speed under similar but non-immersed conditions as in the rest of the study. We then asked participants to fill in the SSQ, to assess their pre-exposure to VR conditions. Successively, we explained how every technique operated.

The order of presentation of the three techniques was counterbalanced. Participants began each trial in a specific location in the physical environment (which was marked accordingly, see Fig. 5). In the VE, this corresponded to a location placed at the entrance of the path. All participants carried a HTC Vive controller in their dominant hand for the duration of each trial. They signalled their readiness to start the trial by pressing the side grip button. After reaching the exit, we invited the participants to take a break and fill in the three questionnaires. Finally, we conducted a brief semistructured interview with each participant, as previously noted.

\subsection{Starting Conditions}

We used the same VE for all trials. The bounds of the Chaperone area (i.e. the area delimited by the grid) were set to a square area of $5 \mathrm{~m} \times 5 \mathrm{~m}$, same as the maximum extents of the tracking area. Participants started from the same physical location (marked by the green circle in Fig. 5) and faced the direction indicated by the arrows (blue for the Space Bender technique, purple for the others).

In the Space Bender technique, participants followed the perimeter of the room in a clockwise direction. In order to maximise the space available in the tracking area, the $100 \mathrm{~m}$ path was programmatically generated as a sequence of $293.5 \mathrm{~m}$ segments (to account for the width of the path when bent), with the last only $1.5 \mathrm{~m}$ long.

In the Stop and Reset and Teleportation techniques, the bending features of the environment were programmatically disabled, whereas the interactive controller features which operated these two techniques were enabled. In these two conditions, with respect to their starting location and orientation, participants walked the length of the Chaperone area. In this case, they could walk for a maximum of $4 \mathrm{~m}$ before the grid appeared. 


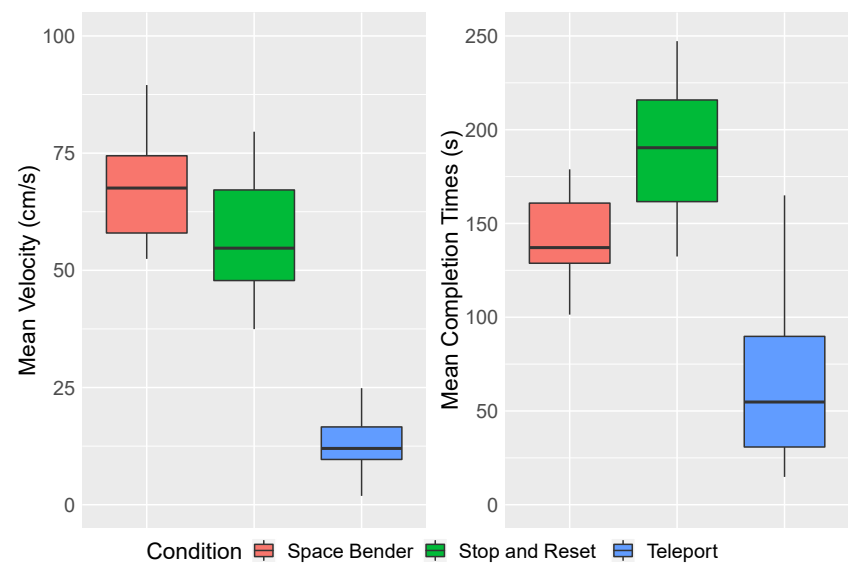

Figure 6: A boxplot of the mean velocity $(\mathrm{cm} / \mathrm{s})$ and completion times (s), grouped by technique.

Table 1: Mean velocity $(\mathrm{cm} / \mathrm{s})$, time $(\mathrm{s})$, and walked distance $(\mathrm{m})$ for each technique.

\begin{tabular}{lrrrrrr}
\hline Technique & Velocity & SD & Time & SD & Length & SD \\
\hline Space Bender & 67.41 & 10.77 & 141.50 & 23.25 & 93.18 & 5.13 \\
Stop and Reset & 57.14 & 12.17 & 197.52 & 43.04 & 108.01 & 2.77 \\
Teleportation & 13.04 & 5.73 & 65.90 & 46.37 & 9.20 & 7.93 \\
\hline
\end{tabular}

In the case of the Stop and Reset technique, although participants could have walked along the diagonal of the room to decrease the number of resets, we observed during pilot testing that participants tended to drift, since they did not walk along a perfect diagonal. This caused a higher variance of the length of each lap than moving along the sides (see Fig. 7b). Therefore, in order to have a more consistent average lap length we opted to have participants walk perpendicularly to the boundaries.

\section{Results}

We logged users' coordinates (taken from the position of the headset) every $20 \mathrm{~ms}$ while using each locomotion technique, our independent variable. The raw logs were first filtered to leave only the measurements between the time they left the initial trigger at the $y-0$ coordinate and the time they left the final trigger which stopped the data logging, and to remove outliers due to sporadic loss of tracking. From this data we calculated our dependent variables: the velocity, the time, the total length of the linear distance effectively walked (see Fig. 6 and Table 1 for the full results), the efficiency and the deviation from the shortest path induced by drifting. We analysed this data with a one-way ANOVA.

In addition, after each trial we asked our participants to fill the SSQ, SUS, and a custom questionnaire. The questionnaire data was analysed with a non-parametric Friedman test.

\subsection{Velocity}

We found a significant effect of technique on participants' walking speeds. Participants walked significantly faster using the Space Bender technique $\left(F_{(2,54)}=160.20, \eta_{p}^{2}=0.86, p<0.01\right)$. Pairwise comparisons (Bonferroni corrected) show that participants using the Space Bender technique walked with significantly faster $(p<0.01)$ average speeds $(M=67.41 \mathrm{~cm} / \mathrm{s}, \operatorname{Max}: 90 \mathrm{~cm} / \mathrm{s}$; see Table 1 for the full results) than while using the Stop and Reset technique $(M=57.14 \mathrm{~cm} / \mathrm{s}, \operatorname{Max}: 80 \mathrm{~cm} / \mathrm{s})$ or the Teleportation technique $(M=13.04 \mathrm{~cm} / \mathrm{s}, \operatorname{Max}: 25 \mathrm{~cm} / \mathrm{s})$. In this latter case, the measured speed refers to the speed of physically walking in the room, without considering the (virtual) displacement occurring from teleportation.
For reference, during the non-immersed initial measurements, participants walked at an average speed of $70.21 \mathrm{~cm} / \mathrm{s}(S D=$ $20.45 \mathrm{~cm} / \mathrm{s}$, Max : $92 \mathrm{~cm} / \mathrm{s}$ ).

\subsection{Completion and Idle Time}

These results are supported by the significant difference in terms of completion times $\left(F_{(2,54)}=54.74, \eta_{p}^{2}=0.67, p<0.01\right)$. Participants using the Teleportation technique preferred relying on the teleportation aspect of the technique over natural walking, and thus reached the exit in significantly shorter times $(M=65.90 \mathrm{~s}, p<$ 0.01 ), than with the two other techniques. However, pairwise comparisons show that the Space Bender technique was significantly faster than the Stop and Reset technique $(p<0.01)$. Participants, on average, completed the task in $141.50 \mathrm{~s}$, nearly a minute less than with the Stop and Reset technique $(M=197.52 \mathrm{~s})$.

We calculated the percentage of time spent barely moving, as estimated by measuring the time when the instantaneous speed was less than $10 \mathrm{~cm} / \mathrm{s}$. We found a significant difference of the technique used $\left(F_{(2,54)}=211.6, \eta_{p}^{2}=0.89, p<0.01\right)$. While using the Stop and Reset, $11.07 \%$ of the time was spent idling, an average of 24.75 s; with the Teleportation, 64.35\% (41.77 s); with the Space Bender, only $2 \%$ (2.82 s).

\subsection{Drifting}

Fig. 7 provides a graphical representation of these behaviours. The Space Bender technique (a) constrained movements within the physical area delimited by the virtual walls, whereas the Stop and Reset technique $(b)$ caused participants to considerably drift in the physical environment after each lap. We calculated the mean shortest distance from the centre of their physical path (i.e. where they should have been if no drifting occurred) and found a significant effect of the technique used $\left(F_{(2,54)}=7.12, \eta_{p}^{2}=0.21, p<0.01\right)$. Pairwise comparisons show that while using the Space Bender participants kept significantly closer $(p<0.01)$ to the centre of their path $(M=24.07 \mathrm{~cm}, S D=7.53 \mathrm{~cm})$ than with the Stop and Reset $(M=67.39 \mathrm{~cm}, S D=50.60 \mathrm{~cm})$ and with the Teleportation $(M=70.57 \mathrm{~cm}, S D=52.81 \mathrm{~cm})$ technique.

\subsection{Walked Distance}

Due to the curvature and length of the path, we found a significant difference $\left(F_{(2,54)}=1669.26, \eta_{p}^{2}=0.98, p<0.01\right)$ in terms of linearly walked distance. With the Teleportation technique, participants tended to walk very little $(M=9.20 \mathrm{~m}$, see Table 1$)$. Fig. 7c shows that participants attempted to combine both walking and teleportation but tended to stop walking when they encountered difficulties in understanding how to teleport and virtually rotate in order to continue walking. Conversely, participants walked significantly more $(p<0.01)$ with the Stop and Reset $(M=108.01 \mathrm{~m})$ than the Space Bender technique $(M=93.18 \mathrm{~m})$.

While logging coordinates from the headset can introduce some additional jitter, observations of participants and their trajectory logs show that on values higher than $100 \mathrm{~m}$, backtracking and not walking perfectly straight had more of an impact. Conversely, values lower than $100 \mathrm{~m}$ while using the Space Bender can be explained by a parallel with the geometry of an athletics track. Indeed, participants who tended to walk between the centre and the outside wall tended to walk more than $100 \mathrm{~m}$, while those who walked closer to the inner wall walked less than that, with the difference accumulating over such a distance. For this reason, we recalculated the times and speeds after each participant had walked a distance equal to the average distance walked with the Space Bender (93.18 m).

The ANOVA and the successive pairwise comparisons confirm that the Space Bender technique is still significantly faster for both walking speed $\left(F_{(2,54)}=156.82, \eta_{p}^{2}=0.85, p<0.01\right)$ and completion times $\left(F_{(2,54)}=55.98, \eta_{p}^{2}=0.67, p<0.01\right)$. The recomputed velocity and times are, Stop and Reset: $57.30 \mathrm{~cm} / \mathrm{s}(S D=12.31 \mathrm{~cm} / \mathrm{s})$, 

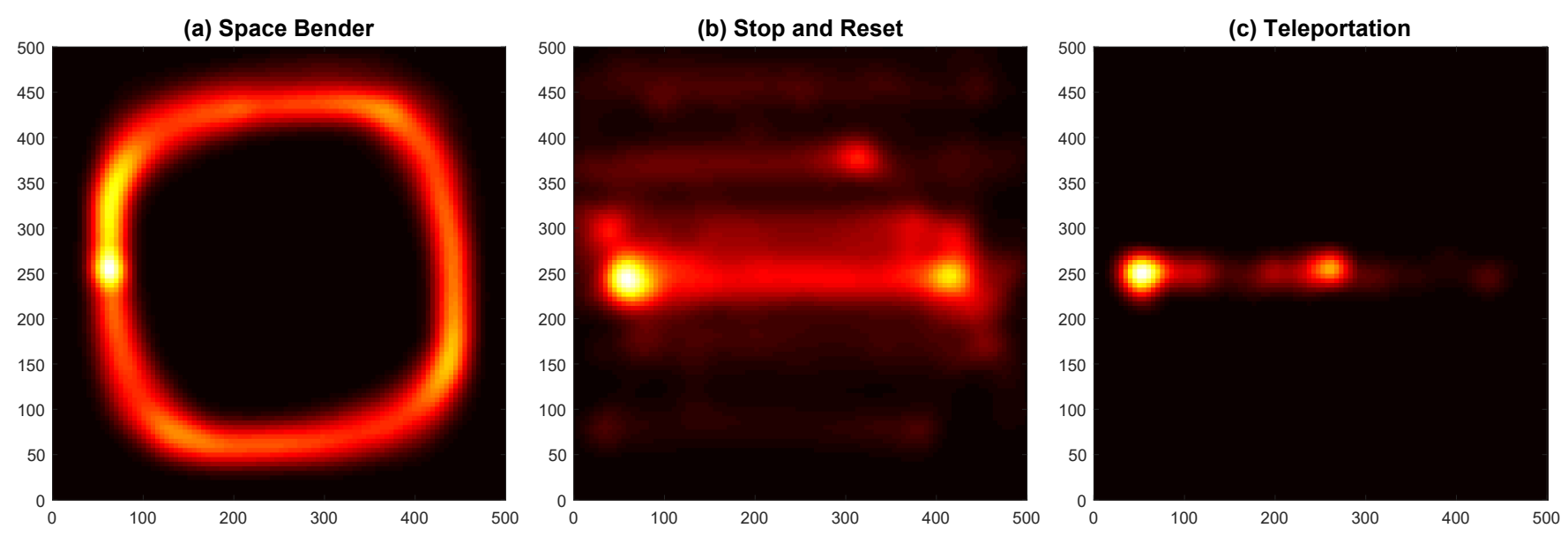

Figure 7: The figure shows the distribution of the participants' trajectories with each technique in the $5 \mathrm{~m} \times 5 \mathrm{~m}$ area.

$183.74 \mathrm{~s}(S D=41.20 \mathrm{~s}) ;$ Teleportation: $12.99 \mathrm{~cm} / \mathrm{s}(S D=6.08 \mathrm{~s})$, $59.05 \mathrm{~s}(S D=43.09 \mathrm{~s}$.

\subsection{Simulator Sickness Questionnaire}

The mean SSQ scores, pre-exposure to VR, average to 3.54 ( $S D=$ 5.50). Although we found a significant difference of technique $\left(\chi^{2}(2)=12.46, p<0.01\right)$, the difference between the two walking techniques is not significant $(p=0.08)$. There was a significant difference between the Teleportation and the Space Bender techniques $(p<0.01)$, which can be explained by the fact that participants were significantly less prone to move and more likely to stand still while using the Teleportation technique (SSQ mean score of $5.51, S D=6.52)$. There were no significant pairwise differences $(p=0.38)$ between Stop and Reset and Teleportation.

The results show that while the average post-exposure SSQ scores for the Space Bender technique are higher than the Stop and Reset (mean scores of 16.53, $S D=16.03$, and 8.86, $S D=8.92$, respectively), in the case of the Space Bender technique they have a higher variance between participants. These scores are in line with the reported values for other locomotion techniques: 24.04 for Redirected Walking [28], 10.2 for Redirected Walking in Place [22], 21.12 in Impossible Spaces [31].

\subsection{Presence and Custom Questionnaire}

We analysed the scores of the SUS presence questionnaire [34] but found no significant differences between the three techniques $(p=0.35)$. The custom questionnaire asked participants to rate their agreements or disagreement with a list of statements, on a one to seven-point scale. These sentences aimed at evaluating their perceived awareness of their position in the physical room, naturalness, comfort, and safety while using each technique.

We found significant differences in terms of Awareness $\left(\chi^{2}(2)=\right.$ $13.58, p<0.01)$, with pairwise comparisons showing that the Space Bender technique $(M=1.95, S D=1.27)$ made participants less aware $(p \leq 0.01)$ of their position in the room, than with the Stop and Reset $(M=4.37, S D=1.8)$ and the Teleportation $(M=4.05, S D=$ 2.27) techniques.

There were significant differences on the reported Naturalness of the techniques $\left(\chi^{2}(2)=17.20, p<0.01\right)$, i.e. how much their virtual movements matched the experience of natural walking. Both walking techniques were unsurprisingly found to be less artificial (Space Bender: $M=4.74, S D=1.74, p<0.01$, Stop and Reset: $M=3.53, S D=1.9, p=0.04)$ than the Teleportation technique $(M=1.89, S D=1.9)$. An explanation for the lower overall scores of the Stop and Reset technique is that participants found the necessity of resetting their orientation a source of breaks in presence [33]. No significant differences were found for Comfort or Safety.

At the end of the study we asked participants to rank the three techniques in order of preference. The majority (11 participants, $57 \%$ ) indicated the Space Bender technique as their first choice, while Stop and Reset was chosen by five (26\%) and Teleportation by three $(16 \%)$. While the Friedman test reported a statistically significant difference in the preference rankings $\left(\chi^{2}(2)=7.68, p=\right.$ $0.02)$, pairwise comparisons only showed a significant difference between Space Bender and Teleportation $(p=0.02)$.

\section{Discussion}

The Space Bender technique aims to provide room-scale VR users with a method to actually walk large distances on linear paths. We think the results of our study satisfy the objectives underpinned by the research questions we had. In the following, we discuss our findings in terms of performance and believability of the experience. We also suggest future research directions and present the limitations of the technique in its current form.

\subsection{Performance}

By definition of Teleportation, the technique unsurprisingly provided the most efficient way of locomotion, resulting in the fastest task completion times. However, it also led participants to move very little. When circumstances allows, there can be compelling reasons to allow users to naturally walk in a VE. Be it to favour immersion [33], promote physical exercising [17], or a more comfortable experience [6]. For room-scale physical environments where natural walking is important or desired, the Space Bender resulted to be the best overall technique to cover large distances in linear VEs. With the Space Bender, users were able to reach the highest mean speeds we measured. Based on the results and our own observations, we think that the minor effort required to follow the $90^{\circ}$ curvature of the bends allowed users to maintain their speed, without coming to a complete halt. We only observed two participants who stopped to change direction in the initial "laps" of the Space Bender technique, whereas the rest turned while just slowing down. In this regard, in the Stop and Reset the necessity of resetting the orientation accumulated over time, as highlighted by the significantly higher percentage of idle time ( $11 \%$ vs $2 \%)$.

Modifying the Stop and Reset to allow $90^{\circ}$ turns instead of $180^{\circ}$, to use more of the physical space available, would increase its complexity as either the user or the system would need to determine along which direction there is physical space available. With a $180^{\circ}$ rotation there is an implicit assumption that the user has space 
to turn back towards the direction along which they came before encountering a physical boundary. Regardless, we think the Space Bender would still be faster as users are significantly less likely to come to a full stop, as is instead the case with Stop and Reset.

Indeed, the seamlessness of the Space Bender technique was cited as its main advantage. During our post-hoc interviews, participant \#9 commented: "It is a more natural way of moving, you can keep walking and walking..." Participant \#5 said: "You could even start running." Given a sufficient safety area around the perimeter and wireless VR support, this might be an interesting direction for future research. Although there were no significant differences in terms of Cybersickness between Stop and Reset and the Space Bender, together with these comments, we view it as a positive result since the results are in line with those from other prior studies [22, 28,31].

Fig. 7 shows that the Space Bender technique is better suited to constrain participants to specific parts of the physical environment as it does not cause the same extent of drifting observed for the Stop and Reset. This can be beneficial if there are physical obstacles in the environment that should be taken into account, either to enhance the believability of the experience [25] or to steer users away [24]. In the trials using the Teleportation technique, we told participants that they should decide whether to walk or teleport. For all but one of our participants, this was the first time using it. We observed some participants who initially attempted to walk until they reached a physical boundary, triggering the appearance of the Chaperone Grid. They attempted to rotate "backwards" so that after reappearing and physically turning, they could have continued to walk in the opposite direction in the real world - forward in the VE - as in the Stop and Reset. However, this behaviour stopped after the first few usages of the Teleportation technique. When asked about the reason, participants cited that in the absence of other constraints they preferred teleporting due to the drastically reduced physical effort required to traverse large distances.

Given that Redirected Walking tends to be noticed in room-scale spaces where it sometimes requires resets [1], the Space Bender could be used to avoid these breaks in presence by bending the VE, when subtle redirection is no longer possible due to space constraints. This overt form of redirection provides more opportunities for users to continue walking, in a more efficient way than other types or resetting controllers.

\subsection{Presence}

By design, the Space Bender technique builds on the assumption that the manipulation will happen in plain sight, without any attempt to hide it. In the words of participant \#8: "[Once] you accept that the environment is morphing, then it is a very immersive experience." Although the SUS questionnaire did not reveal any correlation between the techniques and the feeling of presence, our custom questionnaire showed that participants were significantly less aware of their position in the room while using the Space Bender technique. Indeed, due to their movement pattern they were often surprised of where they found themselves after removing the headset. This might be an indication that the user is less concerned with the outside environment on a cognitive level.

In this user study we did not focus on the issue of the spatial distortion that might arise when more detailed textures are applied to the "bendable" 3D asset or when smaller assets are also present in the scene. As discussed in the Related Work section, Sun et al. evaluated a rendering algorithm that "warps" the VE to accommodate the physical space available and allow users to walk without risking collisions with physical boundaries or obstacles [32]. In their implementation, the VE is presented in various degrees of warping at all times. Although the sample size used in their evaluation is relatively low (seven users), the spatial deformation did not affect the fidelity of the VR experience (six out of seven users graded it at or above $80 \%$ ). Whereas, as previously described, in the Space
Bender technique the distortion is only temporary: the VE will return to its unbent state as soon as the user has walked past the bent segment. Furthermore, when leaving the bent segment, the user will be looking at the VE in its regular state, as the "straightening" happens behind them.

Concerning the Stop and Reset technique, all participants experienced some issues with the Chaperone Grid. During the interviews, and according to our observations, we identified two behaviours. Participants who walked with a more leisurely pace were not likely to be surprised when the grid appeared. Whereas, those who walked faster felt that the walking distance (a maximum of $4 \mathrm{~m}$ ) was enough to gain a momentum, forgetting momentarily about the existence of physical boundaries. Thus, participants had to abruptly stop whenever the grid appeared. Participant \#13 commented: "You are walking and then all of a sudden the grid jumps on your face. I sometimes forgot that it was there."

\subsection{Future work}

The Space Bender, as introduced in this paper, can be applied without modifications to any path that connects two locations without intersections. Going forward, we think it can be extended to enable natural locomotion in more elaborated VEs, by determining the angle of the bend dynamically.

If we imagine the common case of a corridor with adjacent rooms on each side, there are two scenarios. Assuming the user has been redirected to walk along the corridor close to the boundary of the physical space like in our experiment, a room on one side of the user would lead towards the center of the physical room and thus towards a walkable area. In the other, it would lead towards the boundary of the room and potentially towards an obstacle.

A solution would be to combine the Space Bender technique with an approach such as Flexible Spaces [37]. For example, the virtual entrance to a room could be dynamically manipulated in an overt way to lead the user along a "U-turn" bend that would have the user exit in the opposite direction and thus again facing the physical center of the room. It could be triggered by interacting on a door or after walking past an entrance. Like in our implementation, these dynamic bends could only be visible in this state for the time in which the bend is active. Objects located in a VE should not present any issue nor require that they themselves be bent. Those in close proximity to a bent wall might appear in a temporary state of collision. This can be solved by mirroring the bend in those objects as well. The issue of rooms larger than the remaining physical space can be mitigated with other approaches (i.e. resizing it to fit the available space, or using the Stop and Reset to divide it in multiple sections, whereas a 1:1 mapping can be applied to all smaller rooms.

We leave to future research the study of bending larger outdoor areas, as well as exploring other types of overt manipulation to the VE, and combining the Space Bender with Redirected Walking as suggested.

\subsection{Limitations}

The Space Bender technique was used in a $5 \mathrm{~m} \times 5 \mathrm{~m}$ area, which is a considerable space requirement, although still below the extents of the area required by implementations of the redirected walking technique to not notice the manipulation (varying between a radius of $6.1 \mathrm{~m}$ [9] and $22 \mathrm{~m} \mathrm{[28]).} \mathrm{We} \mathrm{evaluated} \mathrm{the} \mathrm{technique} \mathrm{in} \mathrm{an} \mathrm{indoor}$ VE, where participants followed the layout of the virtual walls. In an outdoor environment, stronger forms of virtual barriers should be used, such as fences [25] or water [24].

It also introduces a minor requirement in the 3D asset modelling workflow, as the "bendable" parts of the geometry need to have enough vertices to allow it to approximate a curve with sufficient detail. However, if additional geometry becomes necessary, it would have a negligible impact on performance, given contemporary polygon count allowances. 
Four users cited as a potential issue the lack of an advance warning of the upcoming bend. We placed the trigger prompting the bend animation at $1.17 \mathrm{~m}$ before the start of the curved segment [26], as soon as the user leaves the trigger area. An alternative visualisation could overlay a transparent version of the path in its "bent" state. Similar to how the Chaperone grid works, the bent path might become progressively more opaque as the user approaches the physical boundary, or trigger the animation if the user continues moving in that direction.

\section{CONCLUSION}

The Space Bender locomotion techniques leverages the overt nature of the redirection to provide an alternative method to navigate large linear distances that are significantly longer than the physical space available to the user. Results show that it was significantly faster than the Stop and Reset and led participants to walk significantly more than with the Teleportation technique. It was also overall preferred by participants, who considered it as an immersive experience.

These findings highlight the potential of overt manipulation of the Virtual Environment as a form of redirection. Its advantages in performance and user acceptance provide a compelling alternative to resetting controllers. Thus, we find the Space Bender as a complementary method to conventional forms of redirection: whenever the physical space is not large enough to support subtle redirected walking, it will eventually require a reset. In those circumstances this overt manipulation of the virtual environment provides users with the benefit of not having to come to a full stop, as it only needs a minor correction in speed and trajectory.

Further, bending the geometry of the virtual environment is but one form of overt redirection. This opens up a novel design space for future research to explore. We aim to follow up this work by designing new locomotion techniques in this space, as well as extending the Space Bender to support larger and more complex scenarios.

\section{RefEREnCES}

[1] M. Azmandian, T. Grechkin, M. T. Bolas, and E. A. Suma. Physical space requirements for redirected walking: How size and shape affect performance. In ICAT-EGVE, pp. 93-100, 2015.

[2] B. Bolte, F. Steinicke, and G. Bruder. The jumper metaphor: an effective navigation technique for immersive display setups. In Proceedings of Virtual Reality International Conference. Laval, France, 2011.

[3] D. Bowman, D. Koller, and L. Hodges. Travel in immersive virtual environments: an evaluation of viewpoint motion control techniques. In Proceedings of IEEE 1997 Annual International Symposium on Virtual Reality, pp. 45-52,. IEEE Comput. Soc. Press, Albuquerque, NM, USA, 1997. doi: 10.1109/VRAIS.1997.583043

[4] E. Bozgeyikli, A. Raij, S. Katkoori, and R. Dubey. Point \& Teleport Locomotion Technique for Virtual Reality. In Proceedings of the 2016 Annual Symposium on Computer-Human Interaction in Play - CHI PLAY '16, pp. 205-216. ACM Press, Austin, Texas, USA, 2016. doi: $10.1145 / 2967934.2968105$

[5] G. Bruder, F. Steinicke, K. Hinrichs, and M. Lappe. Reorientation during body turns. In Proceedings of the 15th Joint Virtual Reality Eurographics Conference on Virtual Environments, pp. 145-152. Eurographics Association, Lyon, France, 2009.

[6] S. S. Chance, F. Gaunet, A. C. Beall, and J. M. Loomis. Locomotion Mode Affects the Updating of Objects Encountered During Travel: The Contribution of Vestibular and Proprioceptive Inputs to Path Integration. Presence: Teleoperators and Virtual Environments, 7(2):168-178, Apr. 1998. doi: $10.1162 / 105474698565659$

[7] R. Cools and A. L. Simeone. Investigating the effect of distractor interactivity for redirected walking in virtual reality. In Symposium on Spatial User Interaction, SUI '19. ACM, 2019. doi: 10.1145/3357251. 3357580

[8] J. Frommel, S. Sonntag, and M. Weber. Effects of controller-based locomotion on player experience in a virtual reality exploration game. In Proceedings of the International Conference on the Foundations of Dig- ital Games - FDG '17, pp. 1-6. ACM Press, Hyannis, Massachusetts, 2017. doi: $10.1145 / 3102071.3102082$

[9] T. Grechkin, J. Thomas, M. Azmandian, M. Bolas, and E. Suma. Revisiting detection thresholds for redirected walking: combining translation and curvature gains. In Proceedings of the ACM Symposium on Applied Perception - SAP '16, pp. 113-120. ACM Press, Anaheim, California, 2016. doi: $10.1145 / 2931002.2931018$

[10] E. R. Hoeg, K. V. Ruder, N. C. Nilsson, R. Nordahl, and S. Serafin. An exploration of input conditions for virtual teleportation. In 2017 IEEE Virtual Reality (VR), pp. 341-342. IEEE, Los Angeles, CA, USA, 2017. doi: 10.1109/VR.2017.7892316

[11] V. Interrante, B. Ries, and L. Anderson. Seven League Boots: A New Metaphor for Augmented Locomotion through Moderately Large Scale Immersive Virtual Environments. In 2007 IEEE Symposium on 3D User Interfaces, pp. 167-170. IEEE, Charlotte, NC, USA, 2007. doi: 10.1109/3DUI.2007.340791

[12] J. Jerald, T. Peck, F. Steinicke, and M. Whitton. Sensitivity to scene motion for phases of head yaws. In Proceedings of the 5th symposium on Applied perception in graphics and visualization - APGV '08, $\mathrm{p}$. 155. ACM Press, Los Angeles, California, 2008. doi: 10.1145/1394281 .1394310

[13] R. S. Kennedy, N. E. Lane, K. S. Berbaum, and M. G. Lilienthal. Simulator Sickness Questionnaire: An Enhanced Method for Quantifying Simulator Sickness. The International Journal of Aviation Psychology, 3(3):203-220, July 1993. doi: 10.1207/s15327108ijap0303_3

[14] E. Langbehn, P. Lubos, G. Bruder, and F. Steinicke. Application of redirected walking in room-scale VR. In 2017 IEEE Virtual Reality (VR), pp. 449-450. IEEE, Los Angeles, CA, USA, 2017. doi: 10. 1109/VR.2017.7892373

[15] E. Langbehn, P. Lubos, G. Bruder, and F. Steinicke. Bending the Curve: Sensitivity to Bending of Curved Paths and Application in Room-Scale VR. IEEE Transactions on Visualization and Computer Graphics, 23(4):1389-1398, Apr. 2017. doi: 10.1109/TVCG.2017.2657220

[16] E. Langbehn, P. Lubos, and F. Steinicke. Evaluation of Locomotion Techniques for Room-Scale VR. Joystick, Teleportation, and Redirected Walking. In Proceedings of the Virtual Reality International Conference (VRIC). Laval, France, 2018.

[17] F. F. Mueller, M. R. Gibbs, and F. Vetere. Taxonomy of exertion games. In Proceedings of the 20th Australasian Conference on ComputerHuman Interaction Designing for Habitus and Habitat - OZCHI '08, p. 263. ACM Press, Cairns, Australia, 2008. doi: 10.1145/1517744. 1517772

[18] C. T. Neth, J. L. Souman, D. Engel, U. Kloos, H. H. Bulthoff, and B. J. Mohler. Velocity-dependent dynamic curvature gain for redirected walking. IEEE Transactions on Visualization and Computer Graphics, 18(7):1041-1052, 2012. doi: 10.1109/TVCG.2011.275

[19] N. C. Nilsson, T. Peck, G. Bruder, E. Hodgson, S. Serafin, M. Whitton, F. Steinicke, and E. S. Rosenberg. 15 Years of Research on Redirected Walking in Immersive Virtual Environments. IEEE Computer Graphics and Applications, 38(2):44-56, Mar. 2018. doi: 10.1109/MCG.2018. 111125628

[20] N. C. Nilsson, S. Serafin, F. Steinicke, and R. Nordahl. Natural Walking in Virtual Reality: A Review. Computers in Entertainment, 16(2):1-22, Apr. 2018. doi: 10.1145/3180658

[21] T. C. Peck, H. Fuchs, and M. C. Whitton. Evaluation of reorientation techniques and distractors for walking in large virtual environments. IEEE Transactions on Visualization and Computer Graphics, 15(3):383-394, June 2009. doi: 10.1109/TVCG.2008.191

[22] S. Razzaque, Z. Kohn, and M. C. Whitton. Redirected Walking. In Eurographics 2001 - Short Presentations. Eurographics Association, 2001. doi: $10.2312 /$ egs. 20011036

[23] M. Rietzler, J. Gugenheimer, T. Hirzle, M. Deubzer, E. Langbehn, and E. Rukzio. Rethinking redirected walking: On the use of curvature gains beyond perceptual limitations and revisiting bending gains. In 2018 IEEE International Symposium on Mixed and Augmented Reality (ISMAR), pp. 115-122. IEEE, 2018

[24] A. L. Simeone, I. Mavridou, and W. Powell. Altering User Movement Behaviour in Virtual Environments. IEEE Transactions on Visualization and Computer Graphics, 23(4):1312-1321, Apr. 2017. doi: 10. 1109/TVCG.2017.2657038 
[25] A. L. Simeone, E. Velloso, and H. Gellersen. Substitutional Reality: Using the Physical Environment to Design Virtual Reality Experiences. In Proceedings of the 33rd Annual ACM Conference on Human Factors in Computing Systems - CHI '15, pp. 3307-3316. ACM Press, Seoul, Republic of Korea, 2015. doi: 10.1145/2702123.2702389

[26] M. N. Sreenivasa, I. Frissen, J. L. Souman, and M. O. Ernst. Walking along curved paths of different angles: the relationship between head and trunk turning. Experimental Brain Research, 191(3):313-320, Nov. 2008. doi: 10.1007/s00221-008-1525-3

[27] F. Steinicke, G. Bruder, K. Hinrichs, and A. Steed. Gradual transitions and their effects on presence and distance estimation. Computers \& Graphics, 34(1):26-33, Feb. 2010. doi: 10.1016/j.cag.2009.12.003

[28] F. Steinicke, G. Bruder, J. Jerald, H. Frenz, and M. Lappe. Estimation of detection thresholds for redirected walking techniques. IEEE Transactions on Visualization and Computer Graphics, 16(1):17-27, Feb. 2010. doi: 10.1109/TVCG.2009.62

[29] E. A. Suma, G. Bruder, F. Steinicke, D. M. Krum, and M. Bolas. A taxonomy for deploying redirection techniques in immersive virtual environments. In 2012 IEEE Virtual Reality (VR), pp. 43-46. IEEE, Costa Mesa, CA, USA, Mar. 2012. doi: 10.1109/VR.2012.6180877

[30] E. A. Suma, S. Clark, D. Krum, S. Finkelstein, M. Bolas, and Z. Warte. Leveraging change blindness for redirection in virtual environments. In 2011 IEEE Virtual Reality Conference, pp. 159-166. IEEE, Singapore, Singapore, Mar. 2011. doi: 10.1109/VR.2011.5759455

[31] E. A. Suma, Z. Lipps, S. Finkelstein, D. M. Krum, and M. Bolas. Impossible spaces: Maximizing natural walking in virtual environments with self-overlapping architecture. IEEE Transactions on Visualization and Computer Graphics, 18(4):555-564, 2012. doi: 10.1109/TVCG. 2012.47

[32] Q. Sun, L.-Y. Wei, and A. Kaufman. Mapping virtual and physical reality. ACM Transactions on Graphics (TOG), 35(4):64, 2016.

[33] M. Usoh, K. Arthur, M. C. Whitton, R. Bastos, A. Steed, M. Slater, and F. P. Brooks. Walking $>$ walking-in-place $>$ flying, in virtual environments. In Proceedings of the 26th annual conference on Computer graphics and interactive techniques - SIGGRAPH '99, pp. 359-364.
ACM Press, 1999. doi: 10.1145/311535.311589

[34] M. Usoh, E. Catena, S. Arman, and M. Slater. Using Presence Questionnaires in Reality. Presence: Teleoperators and Virtual Environments, 9(5):497-503, Oct. 2000. doi: 10.1162/105474600566989

[35] K. Vasylevska and H. Kaufmann. Influence of path complexity on spatial overlap perception in virtual environments. In Proceedings of the 25th International Conference on Artificial Reality and Telexistence and 20th Eurographics Symposium on Virtual Environments, pp. 159166. Eurographics Association, Kyoto, Japan, 2015.

[36] K. Vasylevska and H. Kaufmann. Towards efficient spatial compression in self-overlapping virtual environments. In 2017 IEEE Symposium on $3 D$ User Interfaces (3DUI), pp. 12-21. IEEE, Los Angeles, CA, USA, 2017.

[37] K. Vasylevska, H. Kaufmann, M. Bolas, and E. A. Suma. Flexible spaces: Dynamic layout generation for infinite walking in virtual environments. In 2013 IEEE Symposium on 3D User Interfaces (3DUI), pp. 39-42. IEEE, Orlando, FL, USA, 2013.

[38] B. Williams, G. Narasimham, T. P. McNamara, T. H. Carr, J. J. Rieser, and B. Bodenheimer. Updating orientation in large virtual environments using scaled translational gain. In Proceedings of the 3rd symposium on Applied perception in graphics and visualization - APGV '06, p. 21. ACM Press, Boston, Massachusetts, 2006. doi: 10.1145/1140491. 1140495

[39] B. Williams, G. Narasimham, B. Rump, T. P. McNamara, T. H. Carr, J. Rieser, and B. Bodenheimer. Exploring large virtual environments with an HMD when physical space is limited. In Proceedings of the 4th symposium on Applied perception in graphics and visualization - $A P G V$ '07, p. 41. ACM Press, Tubingen, Germany, 2007. doi: 10. $1145 / 1272582.1272590$

[40] R. Yu, W. S. Lages, M. Nabiyouni, B. Ray, N. Kondur, V. Chandrashekar, and D. A. Bowman. Bookshelf and Bird: Enabling real walking in large VR spaces. In 2017 IEEE Symposium on 3D User Interfaces (3DUI), pp. 116-119. IEEE, Los Angeles, CA, USA, 2017. 